\title{
Novel imaging and clinical phenotypes of CONDSIAS disorder caused by a homozygous frameshift variant of ADPRHL2: a case report
}

Hajar Aryan ${ }^{1,2 \dagger}$, Ehsan Razmara ${ }^{3 \dagger}$, Dariush Farhud ${ }^{2,4,5}$, Marjan Zarif-Yeganeh ${ }^{2,6}$, Shaghayegh Zokaei ${ }^{2,7}$, Seyed Abbas Hassani ${ }^{8}$, Mahmoud Reza Ashrafi ${ }^{9}$, Masoud Garshasbi ${ }^{3}$ and Ali Reza Tavasoli ${ }^{*}$

\begin{abstract}
Background: Stress-induced childhood-onset neurodegeneration with variable ataxia and seizures (CONDSIAS) is an autosomal recessive disorder caused by defects in the ADP-Ribosylhydrolase Like 2 (ADPRHL2; OMIM: 618170) gene. This gene encodes the ADP-ribosylhydrolase enzyme (ARH3) that eliminates the addition of poly-ADP ribose (PAR) in the cellular stress onto proteins in the ADP-ribosylation process in which adding one or more ADP-ribose moieties onto the target proteins in the post-translational modification have occurred. In this study, we report a new case of CONDSIAS in the Iranian population. A literature review of CONDSIAS is also included.
\end{abstract}

Case presentation: A four-year-old female patient, born to a consanguineous Iranian family, was referred with various clinical symptoms including impaired speech, variable ataxia, infrequent seizures, and gradual onset of truncal hypotonia. Over time, she developed complete motor and speech regression, bilateral sensorineural hearing loss, infrequent seizures, abdominal distension and gastrointestinal (Gl) intolerance, and loss of consciousness. To better molecularly diagnose, trio-whole-exome sequencing (WES) was performed on the proband and her parents. Sanger sequencing was also applied to investigate co-segregation analysis. Using in silico predictive tools, the possible impacts of the variant on the structure and function of ADPRHL2 protein were predicted. All basic metabolic tests were normal, while serial coronal magnetic resonance imaging (MRI) showed progressive cerebral and cerebellar atrophy in addition to cerebral white matter signal changes as a novel neuroimaging finding. Gl intolerance was another novelty of clinical scenarios in the patient. An auditory brainstem response test showed a severe bilateral sensorineural hearing loss. An electroencephalogram also confirmed focal seizures. From the molecular perspective, a novel homozygous frameshift variant in the ADPRHL2 gene (NM_017825.2; c.636_639del, p.(Leu212fs)) was identified by WES.

Conclusions: CONDSIAS is an ultra-rare neurodegenerative disorder. In the present study, we introduced extraneurological and neuroimaging findings of this disorder in a female child caused by a novel frameshift variation in the ADPRHL2 gene.

Keywords: ADP-ribosylhydrolase, CONDSIAS, Neurodegeneration disease, Novel phenotypes

\footnotetext{
* Correspondence: a_tavasoli@sina.tums.ac.ir

${ }^{+} \mathrm{HA}$ and ER have an equal contribution as the first author

${ }^{9}$ Myelin Disorders Clinic, Pediatric Neurology Division, Children's Medical

Center, Pediatrics Center of Excellence, Tehran University of Medical Sciences,

Tehran, Iran

Full list of author information is available at the end of the article
}

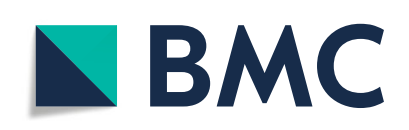

(- The Author(s). 2020 Open Access This article is licensed under a Creative Commons Attribution 4.0 International License, which permits use, sharing, adaptation, distribution and reproduction in any medium or format, as long as you give appropriate credit to the original author(s) and the source, provide a link to the Creative Commons licence, and indicate if changes were made. The images or other third party material in this article are included in the article's Creative Commons licence, unless indicated otherwise in a credit line to the material. If material is not included in the article's Creative Commons licence and your intended use is not permitted by statutory regulation or exceeds the permitted use, you will need to obtain permission directly from the copyright holder. To view a copy of this licence, visit http://creativecommons.org/licenses/by/4.0/. The Creative Commons Public Domain Dedication waiver (http://creativecommons.org/publicdomain/zero/1.0/) applies to the data made available in this article, unless otherwise stated in a credit line to the data. 


\section{Background}

ADP-ribosylation is an important post-translational modification in many physiological pathways, e.g. DNA repair, transcription, translation, and apoptosis [1-4]. In this post-translational modification, the ADP-ribose units are transported from nicotinamide adenine dinucleotide $\left(\mathrm{NAD}^{+}\right)$onto the target protein by ADPribosyltransferases and added to a poly-ADP-ribose (PAR) sequence; this process was firstly suggested by Chambon et al. in 1963 [5, 6]. It was demonstrated that the poly-ADP-ribose levels may be increased 10- to 500folds in response to genotoxic stress, oxidative stress, or mitogenic stimuli [7]. This can prevent cell death in the cellular stress settings, although its over-accumulation, in turn, leads to cell death. To address this, the ADPR HL2 (ADP-Ribosylhydrolase like 2; NM_017825.2; MIM: 610624) gene containing 6 coding exons encodes an ADP-ribosylhydrolase enzyme [8]. This enzyme eliminates the proteins' post-translational addition of polyADP ribose (PAR) in cellular stress. Loss-of-function mutations in the ADPRHL2 gene result in a recently defined disorder called stress-induced childhood-onset neurodegeneration with variable ataxia and seizures (CONDSIAS; OMIM: 618170) [9].

The CONDSIAS is an autosomal recessive disorder which its pertinent gene (ADPRHL2) is mapped on chromosome 1p35.3-p34.1. One frameshift, two nonsense, and three missense mutations have been reported in the association of this gene [10]. The phenotypes of this disorder have been reported as neurodegeneration, variable ataxia and seizures, tremor, nystagmus, balance problems, cerebellar, spinal cord and cerebral atrophy, hearing impairment and occasionally hearing loss, ptosis, ophthalmoplegia, dysarthria, muscle weakness, axonal neuropathy, dysmetria, and tongue fasciculation [10]. Symptoms and severity of the disorder appear to be different in patients and sometimes lead to early childhood death. In other words, although older patients present most of the above-mentioned symptoms, younger patients experience loss of developmental milestones and death in their early infancy [10].

In this study, we introduced a novel homozygous variant, c.636_639del: p.(Leu212fs), in a consanguineous Iranian family, which is associated with CONDSIAS disorder. Besides, novel imaging and clinical features are reported in this study. Future investigations, e.g. doing functional analysis, are necessary to validate the kinds of conclusions that can be drawn from this study.

\section{Case presentation}

The proband (IV.1) is a 4-year-old girl born to a consanguineous Iranian family (Fig. 1a) and was referred to the Children's Medical Center hospital, Tehran, Iran, with different evident clinical symptoms including the new onset of general weakness, gait problem and variable ataxia, impaired speech, focal seizures, and progressive truncal hypotonia. All of the patient's clinical information and the medical histories were collected at the Pediatric Neurology Division, Children's Medical Center, Tehran University of Medical Sciences, Tehran, Iran.

The proband was born to an uneventful cesarean section with the birth-time weight of $3600 \mathrm{~g}$. The birth-time measured head circumference was reported as normal. She was the first child of the family, the second child (IV.2), on the other hand, was completely normal. Family history was also negative for any diseases with similar phenotypes.

Following the tonsillectomy surgery, the proband gradually developed head nodding, upper limbs abnormal movements especially chorea, and then truncal hypotonia. She was also suffering from abdominal distention and food intolerance. Down the line of the surgery, she was referred to the hospital due to neurological deterioration. The early physical and neurological examination of the patient revealed a normal level of consciousness, normal eye contact but extraocular eye movements without any meaningful words production, truncal hypotonia with normal deep tendon reflexes, and weak gag reflexes. Feeding was conducted through nasogastric intubation (NG tube) at initial days of admission but gradually gastrointestinal (GI) intolerance of the patient became detectable and it was also found that she was suffering from severe abdominal distension. The seizure was controlled by administrating proper anti-seizure medications. Basic metabolic tests including serum ammonia, lactate, thyroid and liver function tests, blood gas analysis, serum amino acid chromatography, tandem mass spectrometry (MS/MS), urine organic acids profile were applied to the patient, however, all results were within normal limits. Because of suspected hearing problems, an auditory brainstem response (ABR) test was performed which revealed a severe bilateral sensorineural hearing loss in the patient, while the same condition had not been mentioned in each parent at all.

As early as the first week after the patient's admission, the first brain magnetic resonance imaging (MRI) showed mild supratentorial and cerebellar atrophy with fine deep white matter signal changes in the posterior area (Fig. 1b; A-J and FLAIR-MRI 1-thin white arrows). Routine cerebrospinal fluid analysis (CSF) and CSF viral PCR for herpes simplex virus (HSV), Varicella zoster virus (VZV), enteroviruses, mumps, rubella, and measles viruses had not reported any abnormality. During the hospitalization, the patient was transferred to the pediatric ICU (PICU) section due to a gradually decreased level of consciousness and also cardiorespiratory problems nothing to say GI intolerance. She was admitted to PICU (more than two months) and intubated owing to a more decreased level of consciousness to the Glasgow Coma Scale (GCS) score 3. Anti-seizure medications were stopped due to concern about brain death 


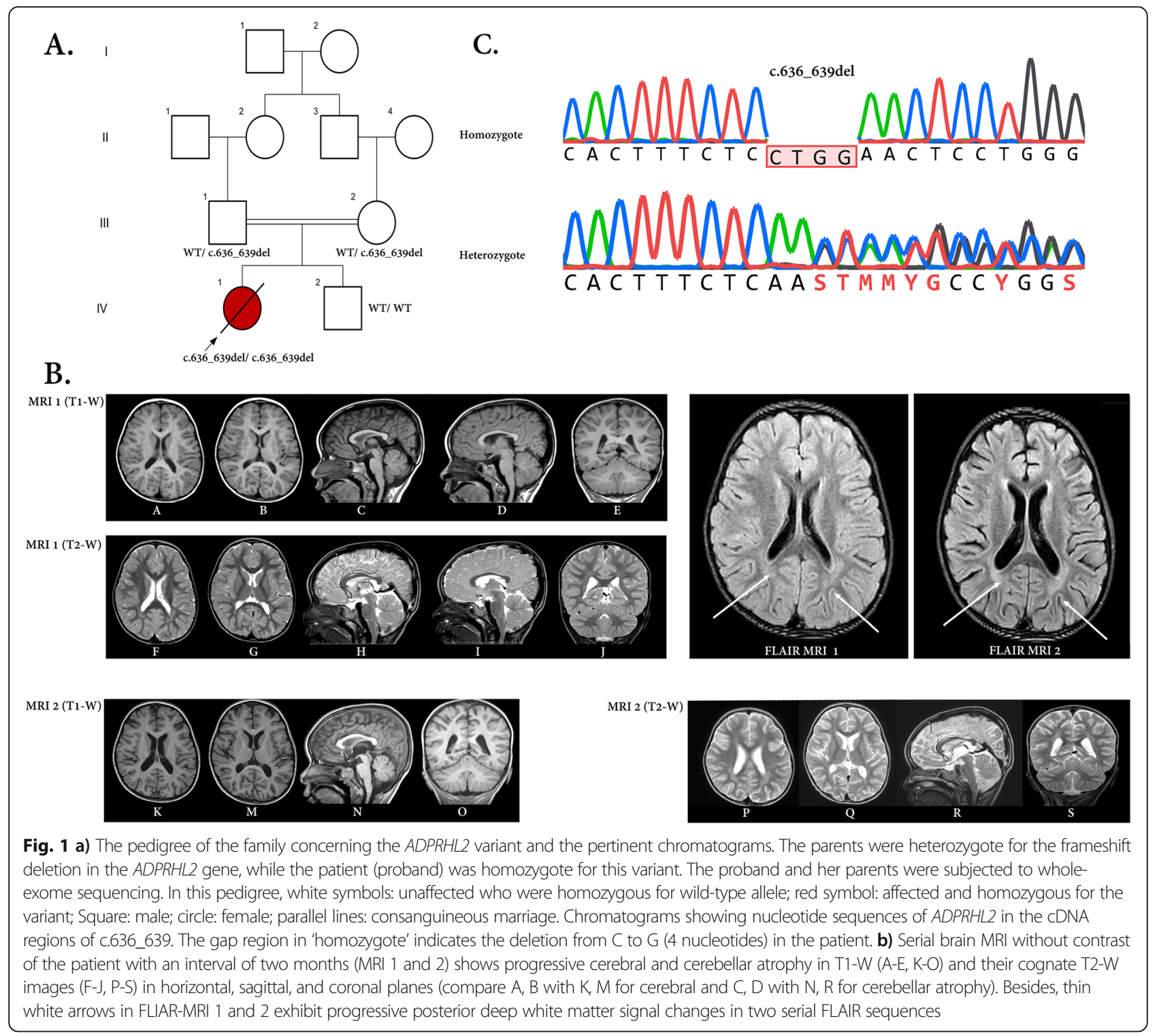

according to EEG monitoring findings. Feeding was stopped through NG-tube due to progressive abdominal distension and total parenteral nutrition (TPN) was conducted to prevent nutritional imbalance. Second brain MRI that was done during PICU admission showed a progressive cerebral and cerebellar atrophy with more prominent deep white matter signal changes in posterior periventricular area (Fig. 1b; K-S and FLAIR-MRI 2-thin white arrows). To achieve an accurate molecular diagnosis, trio-wholeexome sequencing (WES) test was performed. Finally, the patient died due to cardiorespiratory arrest.

\section{Whole exome and sanger sequencing}

For WES, a parent-offspring trio approach was used as previously described [11] with an average coverage depth of 100X on Illumina Hiseq 4000 (Supplementary Method 1).
To begin with, since the pattern of the pedigree was compatible with an autosomal recessive mode of inheritance, only variants that were heterozygous in unaffected parents and homozygous in the proband were considered. The WES data analysis was performed according to the previously reported works [12]. To be on the safe side, the analysis was applied according to the autosomal dominant mode of inheritance too.

Sanger sequencing in forward and reverse directions was performed to validate the candidate variant from WES and then co-segregation analysis was applied to the family. The primers were designed by Primer3.0 webbased server [13] to amplify the exon 4 of the ADPRHL2 gene as follows: Forward: 5-TCTGTCTCCCCTTCTGTT CC-3́ and Reverse: 5-AGGGTCTGCAATTGAGGAAG-3́. The polymerase chain reaction (PCR) was performed in a 
standard condition [14]. The PCR products were sequenced by ABI 3500xl DNA Analyzer (Applied Biosystems Co., Foster City, CA), using the conventional capillary system; sequences were analyzed by the GenomeCompiler online tool (Twist Bioscience, USA) and Mutation Surveyor (v.3.24, SoftGenetics) [15] to identify any alternations (Fig. 1c).

Various databases were utilized to predict the overall score of pathogenicity of the variant, e.g. MutationTaster [16], ENTPRISE-X [17], Polyphen-2 [18], and Sorting Intolerant From Tolerant (SIFT) [19] (Supplementary Method 1). All tools used and also the achieved results are summarized in Table 1. The ConSurf [20] and UCSC databases were also used to provide an evolutionary conservation profile for ADPRHL2 in both protein and gene sequence levels to better judge the potential disrupting role of the variant (Fig. 2a and b). The frequency of the variant was checked in the same ethnic group using the Iranome (http://www.iranome.ir/) [21] as the local database.

\section{Molecular findings}

To show the underlying genetic cause(s), genomic DNA samples were analyzed by trio-WES and as a result, c.636 639del; p.(Leu212fs) homozygous frameshift deletion was confirmed by Sanger sequencing in the fourth exon of the ADPRHL2 gene (Fig. 2a). The identified missense variant was not encountered in dbSNP version 147 [22], 1000 genome project phase 3 [23], Exome Sequencing Project (ESP) [24], ExAC [25], Iranome, Kaviar [26], Human Gene Mutation Database (HGMD) [27], and ClinVar database [28]. This variant was not found in the literature as well.

Finally, we reclassified the variant based on the American College of Medical Genetics and GenomicsAssociation for Molecular Pathology (ACMG-AMP guidelines [29]) into the variant of uncertain significance. Besides, by considering autosomal dominant inheritance, no candidate variant was resulted (Fig. 2c). The novel variant has been deposited to Leiden Open Variation Database (LOVD; https://databases.lovd.nl/shared/individuals/002 99639) [30] and also ClinVar database (Accession Number: SCV001244198).

Several databases such as SIFT, Polyphen-2, and MutationTaster were used to evaluate the possible pathogenicity of the variant. All detailed results are described in Table 1. Furthermore, the conservation study by the ConSurf server showed an average conservation score (Average Score: 5.1) for the involved residues (212369). UCSC genome browser [31] was applied to check the conservation of the region at the nucleotide level. The result showed that the region is highly conserved, especially in primates. Amino acid and nucleotide alignments of ARH3 among higher vertebrates are depicted in Fig. 2b.

\section{Discussion and conclusions}

ADPRHL2 (MIM: 610624; Gene ID: 54936) contains six coding exons that yield to a single protein-coding transcript, ADP-ribosylhydrolase 3 (ARH3) (Fig. 2a). The encoded protein is predicted to have a mitochondrial localization sequence (MTS) and a single enzymatic ADP-ribosyl-glycohydrolase (GH) domain (Fig. 3). This enzyme has been suggested playing an essential role in the cellular stress response pathway, e.g. DNA repair, transcription, telomere function, and apoptosis [32], and adding a poly-ADP-ribose (PAR) [33] to the target proteins. Hence, ADP-ribosylation can regulate protein function in a reversible post-translational modification. In humans, several ADP-ribose transferases, among them, ARH3 looms largely, transfer ADP-ribose from $\mathrm{NAD}^{+}$to target proteins [34].

Mueller-Dieckmann et al. reported the three-dimensional structure of human ADP-ribosylhydrolase 3 (hARH3) and also suggested that its reversible activity is owing to the archetype of an all-alpha-helical protein fold [35]. The PARylated proteins can subsequently initiate cellular stress response pathways and after the resolution of the original insult, ADP-ribose polymers are rapidly removed (Fig. 3). Nevertheless, PAR modification can protect the cell from death [32], excessive PAR accumulation and/or failure to reverse PAR modification can pose the cell in danger of cell-death [36]. Humans have two genes encoding ubiquitously expressed PAR-degrading enzymes: ADPRHL2 and PARG (MIM: 603501). Both can hydrolyze glycosidic bonds between ADP-ribose moieties and prevent PAR accumulation. It seems that inactivating mutations in both genes can increase the axonal cell-death [33], probably leading to white matter changes observed in the patient. Additionally, it has been identified that mitochondrial dysfunction can cause white matter damage/loss in patients [37], but the molecular mechanisms are still unclear.

As discussed, CONDSIAS is an autosomal recessive disorder caused by disruptive mutations in the $A D P R$ $H L 2$ gene. For instance, six independent families carrying ADPRHL2 mutations were reported [10] in them the exome sequencing identified different homozygous mutations including c.530C $>\mathrm{T}$ (exon 4), 5-bp deletion (exon 3), c.1000C $>\mathrm{T}$ (exon 6), c.235A $>\mathrm{C}$ (exon 2),

Table 1 Several online databases used to predict the pathogenicity of p.(Leu212fs) in the family

\begin{tabular}{|c|c|c|c|c|c|c|c|c|c|c|c|}
\hline Gene & NM & Exon & Alternation & $\mathrm{dbSNP}$ & MutationTaster & ENTPRISE-X & Provean & Polyphen & EXAC & 1 k Genome & Iranome \\
\hline ADPRHL2 & NM_017825.2 & 4 & c.636_639del; p.(Leu212fs) & NR & $\mathrm{D}$ & $\mathrm{D}$ & NA & $\mathrm{D}$ & N.R & N.R & N.R \\
\hline
\end{tabular}




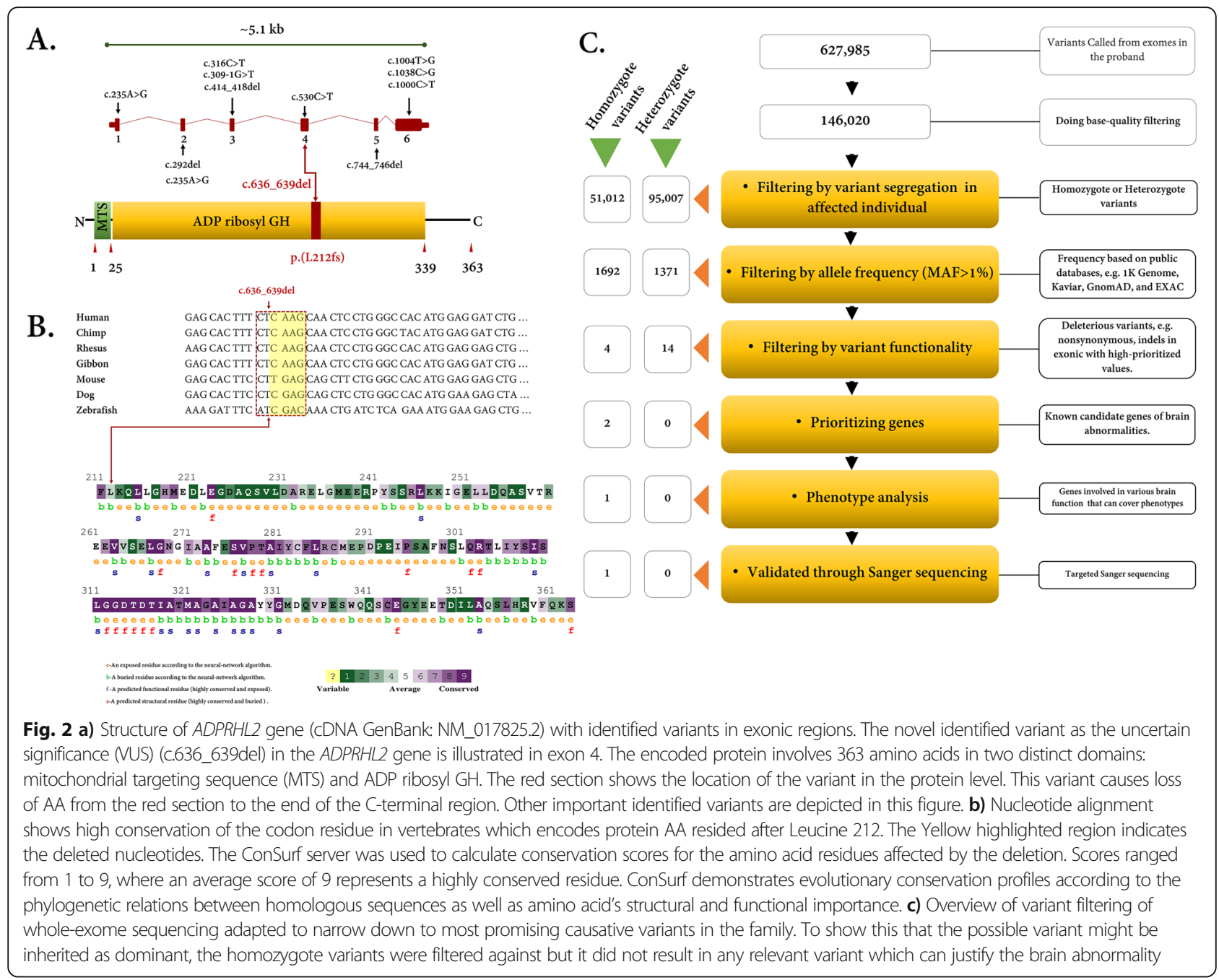

c.100G > A (exon 1), and c.316C $>\mathrm{T}$ (exon 3) in the ADPRHL2 gene (Table 2). All patients showed CONDSIAS common phenotypes. In 2018, Danhauser et al. also detected CONDSIAS disorder in 12 patients. The main reported clinical symptoms in those patients were a developmental delay, intellectual impairment, gait abnormalities, seizures, ataxia, neuropathy, sensorineural hearing loss, microcephaly, and respiratory insufficiency [9] (Table 2). Our case showed the related symptoms are in the line of Danhauser et al. report.

In our case, we found a homozygous frameshift variant in the ADPRHL2 gene (c.636_639del: p.(Leu212fs)) which has not been reported in pieces of literature. We localized this variant to an $\alpha$-helical loop between 11th and 13th $\alpha$ helixes within the ADP-ribosylhydrolase domain which takes a center stage in forming the substrate-binding site, defined by the position of two $\mathrm{Mg}^{2+}$ ions located in adjacent binding sites; therefore, the variant is predicted to affect the protein structure and its enzymatic activity as well (Fig. 4 ae). In other words, the variation identified in this study causes a premature termination codon (246th codon) in the 4th exon of ADPRHL2. It, therefore, seems probable that an altered protein will be expressed but with the truncated Cterminal region which has a detrimental effect on enzyme activity. The phenotype observed may, thus, be a result of the loss of function due to the altered protein inhibiting the enzyme activity of ARH3. This variant was considered a strong candidate for the pathogenic variant in this family.

Medical assessment of the patient, 4-year-old girl, revealed various symptoms including variable ataxia and seizures, impaired speech, and bilateral sensorineural hearing loss. These findings were consistent with previously reported cases in that the most common features were encompassing normal or delayed development, intellectual disability, progressive motor and speech regression, muscle weakness, ataxia and gait problem tongue fasciculation, seizures, sensorineural hearing loss, and neuropathy. Some other extra-neurologic symptoms are respiratory insufficiency, asthma, and ophthalmologic problems. 


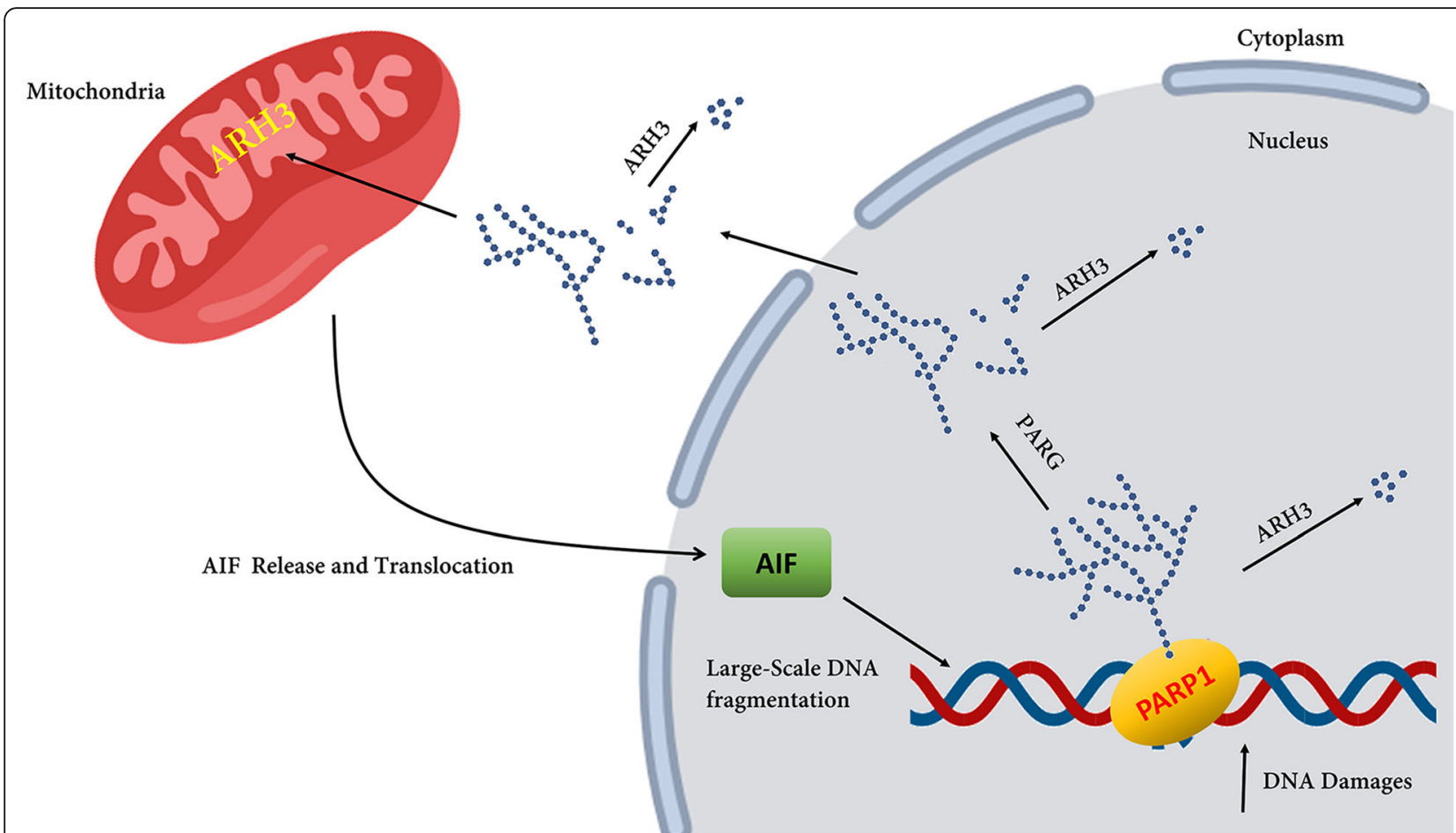

Fig. 3 Model for the role of ARH3 (encoded by the ADPRHL2 gene) in PAR degradation and apoptosis-inducing factor-mediated cell death. The PARP1 activation triggered by DNA damage leads to poly-ADP ribosylation of PARP1 and other acceptor proteins in the nucleus. Poly (ADPribose) glycohydrolase (PARG) hydrolyzes PAR added to the target protein, e.g. PARP1, hence facilitating the protein's translocation to the cytoplasm and mitochondria. ARH3 hydrolyzes PAR. In the nucleus, AIF recruits various nucleases, e.g. cyclophilin A and H2AX, leading to largescale DNA fragmentation. There is a general belief that ARH3 is also located in the mitochondrial matrix. The figure is redrawn from [32]

Severe gastrointestinal intolerance (GI) and abdominal distension was a novel extra-neurologic finding in IV.1 so that she could not be able to tolerate even minimal amounts of liquid diet during admission at PICU, therefore nutrition of the patient was continued through total parenteral nutrition (TPN). According to previous studies, it sounds that hypoactivation of ADPRHL2 has similar effects on the mitochondria as PARP1 hyperactivation. GI intolerance has been reported as a common manifestation of mitochondrial-based disorders [38]. Furthermore, PARP1 hyperactivation (accumulated PAR) has been reported to tightly associated with inflammation in the gastrointestinal system. PARP-1 ${ }^{-/-}$animals displayed a significantly lower level of intestinal inflammation [39]. In this study, we hypothesized that the c.636_639del mutation could be categorized as the loss-of-function mutation, which potentially leads to PAR accumulation. Thus, gastrointestinal manifestation could be probable. Clinically, to find the probable cause of GI intolerance in the patient, we carried out the upper GI endoscopy, and also performed common gastroenterological tests including stool $\mathrm{PH}$ test, faecal (fecal) calprotectin, and routine microbiology tests. None of them showed any abnormalities.

The most common neuroimaging findings that have been stated in association with CONDIAS disorder are cerebral and cerebellar atrophy, progressive cerebellar vermis atrophy, basal ganglia, corpus callosum, and spinal cord involvement but cerebral white matter signal changes have not been reported. Therefore, another phenotypical novelty of the patient was progressive deep white matter signal changes in the occipital area of the brain which has not been reported in neuroimaging findings of CONDIAS disorder. To impute the white matter changes to the mutation in ADPRHL2, we excluded other possible causes, e.g. circulatory changes, any possible systemic hypotension during and/or after hypoxia (asphyxia), a positive history of infection (inflammation), and mechanical problems during delivery resulting in excessive molding and depression of the skull. Thus, to our knowledge, this study extends both clinical and neuroimaging findings of this disorder.

The detection of an ADPRHL2 variation in this family asserts that the ARH3 plays an important role in the brain. This study also demonstrates the effectiveness of WES technology for detecting causative gene defects. In this study, a frameshift deletion at the important part of ARH3 protein, ADP ribosyl GH domain, was introduced as a cause of CONDIAS. The phenotypes observed were consistent with the reported cases, however, we detected some novel phenotypes as well, e.g. progressive deep white matter signal changes in the occipital area and cerebral white matter of 


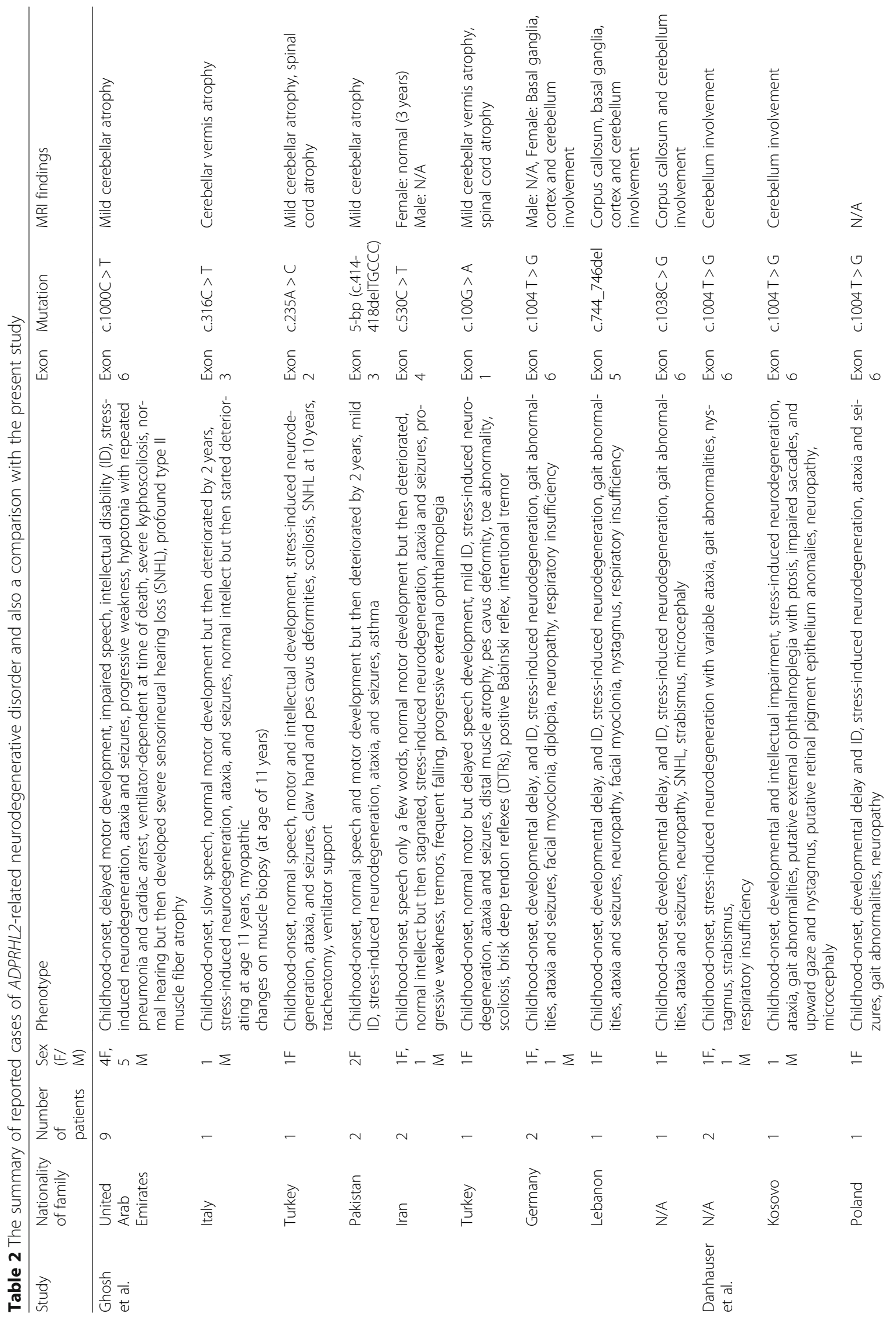




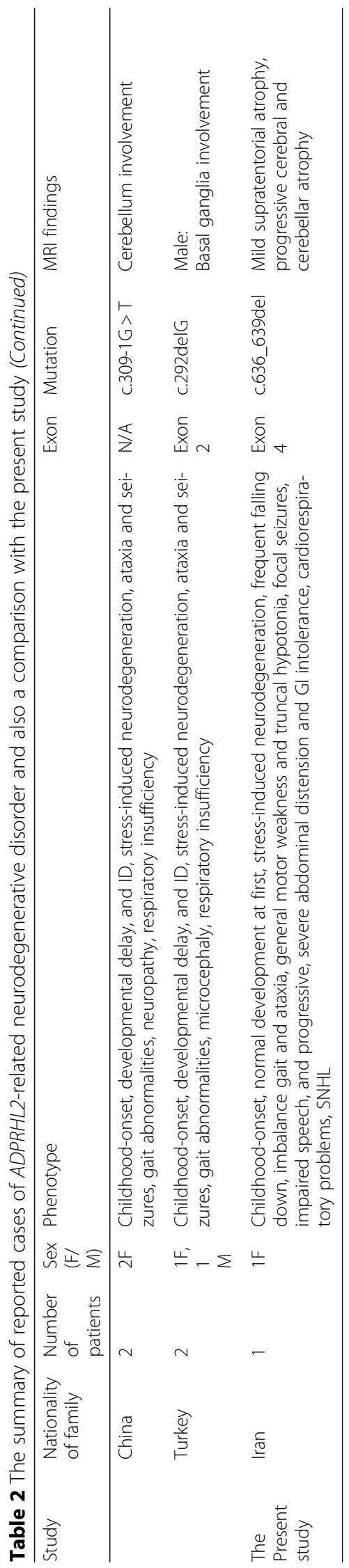




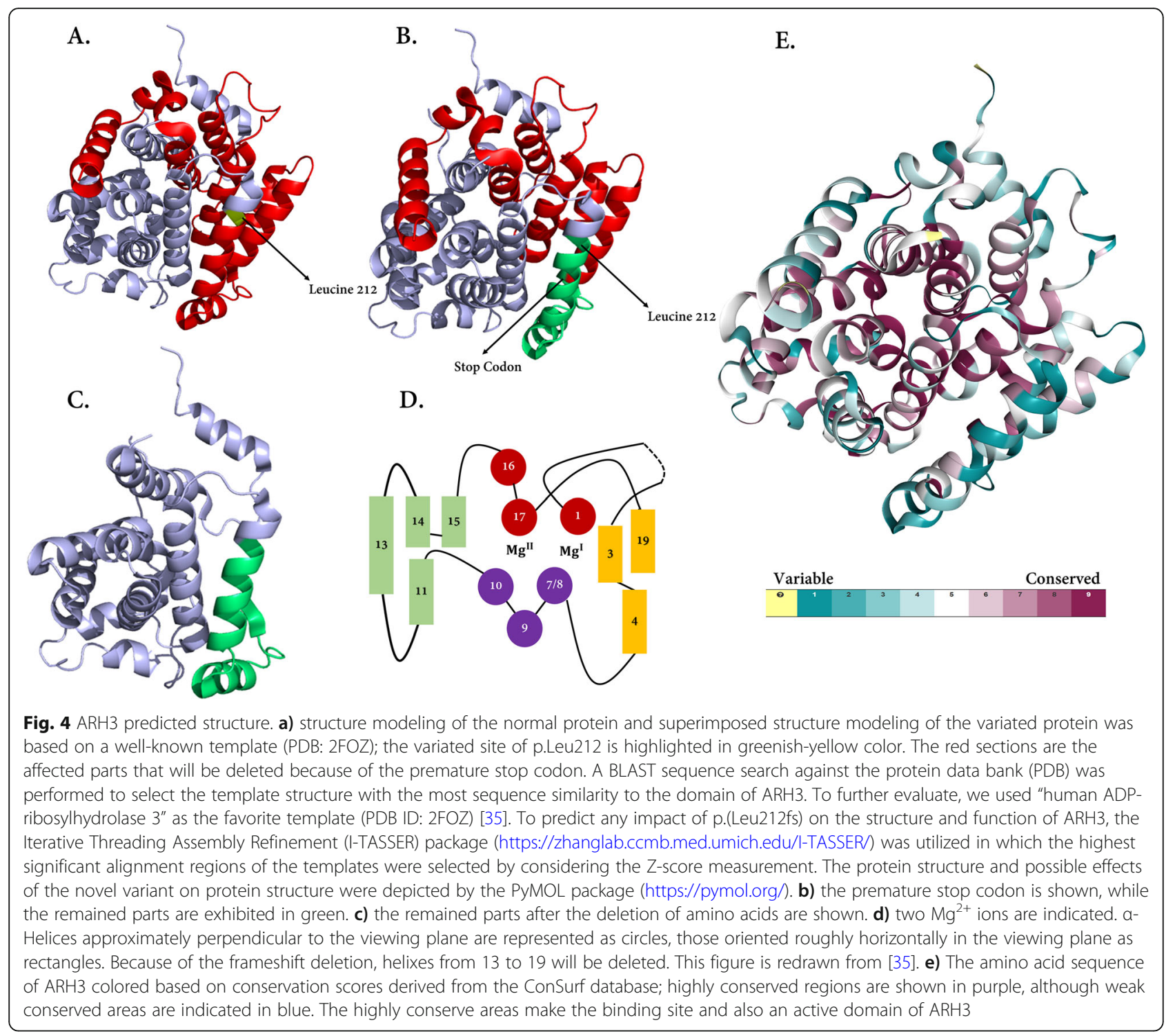

the brain, gastrointestinal intolerance, and abdominal distension. Future investigations, i.e. doing functional analysis, are necessary to validate some kind of conclusions drawn from this study.

In a nutshell, the present study underscores the usefulness of WES in finding the genetic basis of neurological disease. The upshot of this is the possibility that the c.636_639del variant in exon 4 of ADPRHL2 can make encoded protein to malfunction. Nevertheless, before using this data in genetic counseling, we strongly suggest doing functional analysis.

\section{Supplementary information}

Supplementary information accompanies this paper at https://doi.org/10. 1186/s12883-020-01873-3.

Additional file 1

\section{Abbreviations}

CONDSIAS: Stress-induced childhood-onset neurodegeneration with variable ataxia and seizures; ADPRHL2: ADP-Ribosylhydrolase Like 2; ARH3: ADPribosylhydrolase enzyme; PAR: Poly-ADP ribose; Gl: Gastrointestinal intolerance; WES: Whole-exome sequencing; NG tube: Nasogastric intubation; PICU: Pediatric ICU; GCS: Glasgow Coma Scale; ESP: Exome Sequencing Project; PDB: Protein data bank; I-TASSER: Iterative Threading Assembly Refinement

\section{Acknowledgments}

We thank all the family members for their participation in this study.

\section{Authors' contributions}

HA and AT conceived and planned the experiments. DF, ER, MZY, and SZ carried out the experiments. MZY, SAH, and SZ contributed to sample preparation. AT, MA and MG contributed to the interpretation of the results. AT, MG, MRA, and ER took the lead in writing the manuscript. All authors provided critical feedback and helped shape the research, analysis, and manuscript. The authors read and approved the final manuscript.

Funding

All authors had no funding regarding this case report. 


\section{Availability of data and materials}

The datasets analysed during the current study are available from the corresponding author on reasonable request. The novel variant has been submitted to the specific gene variant database Leiden Open Variation Database (LOVD; individual number: 00299639; https://databases.lovd.nl/ shared/individuals/00299639) and ClinVar database (accession number: SCV001244198; https://www.ncbi.nlm.nih.gov/clinvar/53619267).

\section{Ethics approval and consent to participate}

The written, informed consent was obtained from all participants or their respective guardians. The study protocol was approved by the local medical ethics committee of Tehran University of Medical Sciences, Tehran, Iran under the ethical code of "IR.TUMS.1396.3082."

\section{Consent for publication}

Consent to publish was obtained in written form from each participant Additionally, the participants consented to the publication of all personal and medical details included in the case report as well as the accompanying images.

\section{Competing interests}

The authors declare that they have no competing interests.

\section{Author details}

${ }^{1}$ National Institute of Genetic Engineering and Biotechnology, Tehran, Iran. ${ }^{2}$ Dr. Farhud's Genetics Clinic, Tehran, Iran. ${ }^{3}$ Department of Medical Genetics, Faculty of Medical Sciences, Tarbiat Modares University, Tehran, Iran. ${ }^{4}$ School of Public Health, Tehran University of Medical Sciences, Tehran, Iran. ${ }^{5}$ Department of Basic Sciences, Iranian Academy of Medical Sciences, Tehran, Iran. ${ }^{6}$ Cellular and Molecular Endocrine Research Center, Research Institute of Endocrine Sciences, Shahid Beheshti University of Medical Sciences, Tehran, Iran. ${ }^{7}$ School of Advanced Medical Science, Islamic Azad University, Tehran, Iran. ${ }^{8}$ Pediatric Intensive Care Medicine Department, Children's Medical Center, Tehran University of Medical Sciences, Tehran, Iran. ${ }^{9}$ Myelin Disorders Clinic, Pediatric Neurology Division, Children's Medical Center, Pediatrics Center of Excellence, Tehran University of Medical Sciences, Tehran, Iran.

\section{Received: 23 April 2020 Accepted: 28 July 2020}

Published online: 03 August 2020

\section{References}

1. Munnur D, Ahel I. Reversible mono-ADP-ribosylation of DNA breaks. FEBS J. 2017;284(23):4002-16. https://doi.org/10.1111/febs.14297.

2. Martinez-Zamudio R, Ha HC. Histone ADP-ribosylation facilitates gene transcription by directly remodeling nucleosomes. Mol Cell Biol. 2012;32(13): 2490-502. https://doi.org/10.1128/MCB.06667-11.

3. Jankevicius G, Ariza A, Ahel M, Ahel I. The toxin-antitoxin system DarTG catalyzes reversible ADP-ribosylation of DNA. Mol Cell. 2016;64(6):1109-16. https://doi.org/10.1016/j.molcel.2016.11.014.

4. Wright SC, Wei QS, Kinder DH, Larrick JW. Biochemical pathways of apoptosis: nicotinamide adenine dinucleotide-deficient cells are resistant to tumor necrosis factor or ultraviolet light activation of the 24-kD apoptotic protease and DNA fragmentation. J Exp Med. 1996;183(2):463-71.

5. Wang Y, Rösner D, Grzywa M, Marx A. Chain-terminating and clickable NAD+ analogues for labeling the target proteins of ADP-Ribosyltransferases. Angew Chem Int Ed. 2014;53(31):8159-62. https://doi.org/10.1002/anie.201404431.

6. Woodhouse BC, Dianov GL. Poly ADP-ribose polymerase-1: an international molecule of mystery. DNA repair. 2008;7(7):1077-86. https://doi.org/10.1016/ j.dnarep.2008.03.009.

7. Zhang P, Maruyama T, Konkel JE, Abbatiello B, Zamarron B, Wang ZQ, et al. PARP-1 controls immunosuppressive function of regulatory $T$ cells by destabilizing Foxp3. PloS one. 2013;8(8):e71590. https://doi.org/10.1371/ journal.pone.0071590.

8. Hoch NC, Polo LM. ADP-ribosylation: from molecular mechanisms to human disease. Genet Mol Biol. 2020;43:1. https://doi.org/10.1590/1678-4685-GMB2019-0075.

9. Danhauser K, Alhaddad B, Makowski C, Piekutowska-Abramczuk D, Syrbe S, Gomez-Ospina N, et al. Bi-allelic ADPRHL2 mutations cause neurodegeneration with developmental delay, ataxia, and axonal neuropathy. Am J Hum Genet. 2018;103(5):817-25. https://doi.org/10.1016/j. ajhg.2018.10.005.
10. Ghosh SG, Becker K, Huang H, Dixon-Salazar T, Chai G, Salpietro V, et al. Biallelic mutations in ADPRHL2, encoding ADP-ribosylhydrolase 3, lead to a degenerative pediatric stress-induced epileptic ataxia syndrome. Am J Hum Genet. 2018;103(3):431-9. https://doi.org/10.1016/j.ajhg.2018.07.010.

11. Binaafar S, Razmara E, Mahdieh N, Sahebjame H, Tavasoli AR, Garshasbi M. A novel missense variant in GPT2 causes non-syndromic autosomal recessive intellectual disability in a consanguineous Iranian family. Eur J Med Genet. 2020;103853. https://doi.org/10.1016/j.ejmg.2020.103853.

12. Razmara E, Azimi H, Bitaraf A, Daneshmand MA, Galehdari M, Dokhanchi M, et al. Whole-exome sequencing identified a novel variant in an Iranian patient affected by pycnodysostosis. Mol Genetics Genomic Med:e1118. https://doi.org/10.1002/mgg3.1118.

13. Rozen S, Skaletsky H. Primer3 on the WWW for general users and for biologist programmers. In: Bioinformatics methods and protocols. Springer; 2000. p. 365-86. https://doi.org/10.1385/1-59259-192-2:365.

14. Esmaeilzadeh-Gharehdaghi E, Razmara E, Bitaraf A, Mahmoudi M, Garshasbi M. S3440P substitution in C-terminal region of human Reelin dramatically impairs secretion of Reelin from HEK 293T cells. Cell Mol Biol (Noisy-legrand). 2019;65(6):12-6.

15. Minton JA, Flanagan SE, Ellard S. Mutation surveyor: software for DNA sequence analysis. In: PCR Mutation Detection Protocols. Springer; 2011. p. 143-53. https://doi.org/10.1007/978-1-60761-947-5_10.

16. Schwarz JM, Rödelsperger C, Schuelke M, Seelow D. MutationTaster evaluates disease-causing potential of sequence alterations. Nature Methods. 2010;7(8):575. https://doi.org/10.1038/nmeth0810-575.

17. Zhou H, Gao M, Skolnick J. ENTPRISE-X: predicting disease-associated frameshift and nonsense mutations. PLoS One. 2018;13:5. https://doi.org/10. 1371/journal.pone.0196849.

18. Adzhubei I, Jordan DM, Sunyaev SR. Predicting functional effect of human missense mutations using PolyPhen-2. Curr Protocols Hum Genetics. 2013: 76(1):7-20. 1-7. 41. https://doi.org/10.1002/0471142905.hg0720s76.

19. $\mathrm{Ng} P$ PC, Henikoff S. SIFT: predicting amino acid changes that affect protein function. Nucleic Acids Res. 2003;31(13):3812-4. https:/doi.org/10.1093/nar/gkg509.

20. Glaser F, Pupko T, Paz I, Bell RE, Bechor-Shental D, Martz E, et al. ConSurf: identification of functional regions in proteins by surface-mapping of phylogenetic information. Bioinformatics. 2003;19(1):163-4. https://doi.org/ 10.1093/bioinformatics/19.1.163.

21. Fattahi Z, Beheshtian M, Mohseni M, Poustchi H, Sellars E, Nezhadi SH, et al. Iranome: a catalog of genomic variations in the Iranian population. Hum Mutat. 2019:40(11):1968-84. https://doi.org/10.1002/humu.23880.

22. Sherry ST, Ward M-H, Kholodov M, Baker J, Phan L, Smigielski EM, et al. dbSNP: the NCBI database of genetic variation. Nucleic Acids Res. 2001; 29(1):308-11. https://doi.org/10.1093/nar/29.1.308.

23. Li H, Handsaker B, Wysoker A, Fennell T, Ruan J, Homer N, et al. 1000 genome project data processing subgroup. 2009. The sequence alignment/ map format and samtools. Bioinformatics. 2009;25(16):2078-9. https://doi. org/10.1093/bioinformatics/btp352.

24. Auer PL, Reiner AP, Wang G, Kang HM, Abecasis GR, Altshuler D, et al. Guidelines for large-scale sequence-based complex trait association studies: lessons learned from the NHLBI exome sequencing project. Am Journal Hum Genet. 2016;99(4):791-801. https://doi.org/10.1016/j.ajhg.2016.08.012.

25. Karczewski K, Weisburd B, Thomas B, Solomonson M, Ruderfer DM, Kavanagh D, et al. The ExAC browser: displaying reference data information from over 60000 exomes. Nucleic Acids Res. 2017;45(D1):D840-D5. https:// doi.org/10.1093/nar/gkw971.

26. Glusman G, Caballero J, Mauldin DE, Hood L, Roach JC. Kaviar: an accessible system for testing SNV novelty. Bioinformatics. 2011;27(22):3216-7. https:// doi.org/10.1093/bioinformatics/btr540.

27. Stenson PD, Ball EV, Mort M, Phillips AD, Shiel JA, Thomas NS, et al. Human gene mutation database (HGMD): 2003 update. Hum Mutat. 2003;21 (6): 577-81. https://doi.org/10.1002/humu.10212.

28. Landrum MJ, Lee JM, Riley GR, Jang W, Rubinstein WS, Church DM, et al. ClinVar: public archive of relationships among sequence variation and human phenotype. Nucleic Acids Res. 2014;42(D1):D980-D5. https://doi.org/ 10.1093/nar/gkt1113.

29. Li Q, Wang K. InterVar: clinical interpretation of genetic variants by the 2015 ACMG-AMP guidelines. Am J Hum Genet. 2017;100(2):267-80. https://doi. org/10.1016/j.ajhg.2017.01.004. 
30. Fokkema IF, Taschner PE, Schaafsma GC, Celli J, Laros JF, den Dunnen JT. LOVD v. 2.0: the next generation in gene variant databases. Hum Mutat. 2011;32(5):557-63. https://doi.org/10.1002/humu.21438.

31. Karolchik D, Baertsch R, Diekhans M, Furey TS, Hinrichs A, Lu Y, et al. The UCSC genome browser database. Nucleic Acids Res. 2003;31(1):51-4. https:// doi.org/10.1093/nar/gkg129.

32. Mashimo M, Kato J, Moss J. ADP-ribosyl-acceptor hydrolase 3 regulates poly (ADP-ribose) degradation and cell death during oxidative stress. Proc Natl Acad Sci. 2013;110(47):18964-9. https://doi.org/10.1073/pnas.1312783110.

33. Andrabi SA, Kim NS, Yu S-W, Wang H, Koh DW, Sasaki M, et al. Poly (ADPribose)(PAR) polymer is a death signal. Proc Natl Acad Sci. 2006;103(48): 18308-13. https://doi.org/10.1073/pnas.0606526103.

34. Koch-Nolte F, Kernstock S, Mueller-Dieckmann C, Weiss MS, Haag F. Mammalian ADP-ribosyltransferases and ADP-ribosylhydrolases. Front Biosci. 2008;13:6716-29. https://doi.org/10.2741/3184.

35. Mueller-Dieckmann C, Kernstock S, Lisurek M, von Kries JP, Haag F, Weiss MS, et al. The structure of human ADP-ribosylhydrolase 3 (ARH3) provides insights into the reversibility of protein ADP-ribosylation. Proc Natl Acad Sci. 2006;103(41):15026-31. https://doi.org/10.1073/pnas.0606762103.

36. Wang Y, Dawson VL, Dawson TM. Poly (ADP-ribose) signals to mitochondrial AlF: a key event in parthanatos. Exp Neurol. 2009;218(2):193-202. https://doi. org/10.1016/j.expneurol.2009.03.020.

37. Gropman AL. Neuroimaging in mitochondrial disorders. Neurotherapeutics. 2013;10(2):273-85. https://doi.org/10.1007/s13311-012-0161-6.

38. Finsterer J, Frank M. Gastrointestinal manifestations of mitochondrial disorders: a systematic review. Ther Adv Gastroenterol. 2017;10(1):142-54. https://doi.org/10.1177/1756283X16666806.

39. Dörsam B, Seiwert N, Foersch S, Stroh S, Nagel G, Begaliew D, et al. PARP-1 protects against colorectal tumor induction, but promotes inflammationdriven colorectal tumor progression. Proc Natl Acad Sci. 2018;115(17): E4061-E70. https://doi.org/10.1073/pnas.1712345115.

\section{Publisher's Note}

Springer Nature remains neutral with regard to jurisdictional claims in published maps and institutional affiliations.

Ready to submit your research? Choose BMC and benefit from:

- fast, convenient online submission

- thorough peer review by experienced researchers in your field

- rapid publication on acceptance

- support for research data, including large and complex data types

- gold Open Access which fosters wider collaboration and increased citations

- maximum visibility for your research: over $100 \mathrm{M}$ website views per year

At BMC, research is always in progress.

Learn more biomedcentral.com/submissions 